\title{
Diacylglycerol Kinase $\zeta$ Attenuates Pressure Overload-Induced Cardiac Hypertrophy
}

\author{
Mutsuo Harada, MD; Yasuchika Takeishi, MD; Takanori Arimoto, MD; \\ Takeshi Niizeki, MD; Tatsuro Kitahara, MD; Kaoru Goto, MD*; \\ Richard A. Walsh, MD**; Isao Kubota, MD
}

\begin{abstract}
Background The Gaq protein-coupled receptor (GPCR) signaling pathway, which includes diacylglycerol (DAG) and protein kinase $\mathrm{C}(\mathrm{PKC})$, plays a critical role in the development of cardiac hypertrophy and heart failure. DAG kinase (DGK) phosphorylates DAG and controls cellular DAG levels, thus acting as a regulator of GPCR signaling. It has been previously reported that DGK inhibited GPCR agonist-induced activation of the DAG-PKC signaling and subsequent cardiomyocyte hypertrophy, so the purpose of this study was to examine whether DGK modifies the development of cardiac hypertrophy induced by pressure overload.

Methods and Results Thoracic transverse aortic constriction (TAC) was created in transgenic mice with cardiac-specific overexpression of DGK (DGK -TG) and wild-type (WT) mice. Increases in heart weight at 4 weeks after TAC were attenuated in DGK -TG mice compared with WT mice. Increases in interventricular septal thickness, dilatation of the left ventricular cavity, and decreases in left ventricular systolic function in WT mice were observed with echocardiography at 4 weeks after TAC surgery. However, these structural and functional changes after TAC were attenuated in DGK马 -TG mice. In WT mice, cardiac fibrosis and up-regulation of profibrotic genes, such as transforming growth factor $\beta$ 1, collagen type I, and collagen type III, were observed at 4 weeks after TAC. However, cardiac fibrosis and gene induction of type I and type III collagens, but not transforming growth factor $\beta$, were blocked in DGK -TG mice.

Conclusion These results are the first in vivo evidence that DGK suppresses cardiac hypertrophy and fibrosis and prevents impaired left ventricular systolic function caused by pressure overload. (Circ J 2007; 71: 276-282)
\end{abstract}

Key Words: Diacylglycerol kinase; Hypertrophy; Protein kinase C; Pressure overload; Remodeling

M yocardial hypertrophy is an adaptive response of the heart to increasing workload such as hypertension, valve disorders, and ischemic events! On the other hand, myocardial hypertrophy also represents an independent risk factor for the development of heart failure and subsequent cardiac death? The signal transduction pathways mediating cardiac hypertrophy have been examined in a variety of in vitro and in vivo studies, 3 and the Gaq protein-coupled receptor (GPCR) signaling pathway that includes protein kinase $\mathrm{C}(\mathrm{PKC})$ plays a critical role in this process. ${ }^{4,5}$ The binding of diacylglycerol (DAG) to the $\mathrm{C} 1$ domain of PKC induces an active conformation, and activated $\mathrm{PKC}$ phosphorylates several myocardial proteins leading to myocardial hypertrophy and congestive heart failure ${ }^{6-9}$

DAG kinase (DGK) is an enzyme that is responsible for controlling cellular levels of DAG by converting it to phosphatidic acid! ${ }^{10-14}$ We have recently demonstrated in cultured rat neonatal cardiomyocytes that adenoviral-mediated overexpression of DGK , the predominant cardiac isoform,

(Received August 17, 2006; revised manuscript received November 2, 2006; accepted November 20, 2006)

Department of Cardiology, Pulmonology and Nephrology, *Department of Anatomy and Cell Biology, Yamagata University School of Medicine, Yamagata, Japan and **Department of Medicine, Case Western Reserve University, Cleveland, OH, USA

Mailing address: Yasuchika Takeishi, MD, Department of Cardiology, Pulmonology, and Nephrology, Yamagata University School of Medicine, 2-2-2 Iida-Nishi, Yamagata 990-9585, Japan. E-mail: takeishi@med.id.yamagata-u.ac.jp blocks endothelin-1-induced increases in protein synthesis concomitant with increases in cell size and reactivation of fetal genes via the inhibition of the PKCe-extracelluler signal regulated kinase-activator protein-1 signaling pathway 15 We have generated transgenic mice with cardiac-specific postnatal overexpression of DGK (DGK -TG) using an a -myosin heavy chain (MHC) promoter and demonstrated that DGKS prevents angiotensin II- and phenylephrineinduced activation of the DAG-PKC downstream signaling cascades and subsequent cardiac hypertrophy by controlling cellular DAG levels $!^{16}$ However, the effects of DGK on mechanical overload-induced activation of intra-cellular signaling and resultant cardiac structural changes have not been previously examined.

In the present study, we tested the hypothesis that DGK马 attenuates cardiac hypertrophy in response to pressure overload. We created a mouse model of pressure overloadinduced cardiac hypertrophy by thoracic transverse aortic constriction (TAC) and compared cardiac morphology, function, cellular signaling, and gene expression between DGKS -TG and wild-type (WT) mice.

\section{Methods}

\section{Animals}

DGK -TG mice were created in our institution as previously reported, ${ }^{16}$ and DGK -TG (line 70) and WT littermate mice aged 8-10 weeks old were used in the present study. Mice were housed in a facility with a 12/12-h light/dark cycle and were given free access to water and standard 
rodent chow. The room was kept specific-pathogen-free. All experimental procedures were performed according to the animal welfare regulations of Yamagata University School of Medicine, and the study protocol was approved by the Animal Subjects Committee of Yamagata University School of Medicine. The investigation conformed to the Guide for the Care and Use of Laboratory Animals published by the US National Institutes of Health.

\section{Thoracic Transverse Aortic Constriction}

TAC was performed as described previously ${ }^{17}$ Briefly, mice $(\approx 20-25 \mathrm{~g}$ body weight) were anesthetized by intraperitoneal injection with a mixture of ketamine $(80 \mathrm{mg} / \mathrm{kg})$ and xylazine $(8 \mathrm{mg} / \mathrm{kg})$. Animals were intubated with a $20-$ gauge polyethylene catheter and ventilated with a rodent ventilator (Harvard Apparatus, Holliston, MA, USA). The chest cavity was opened at the second intercostal space at the left upper sternal border. The transverse section of the aorta was freed, an 8-0 prolene suture was passed around the aorta between the right innominate and left common carotid arteries, a tight ligature was tied against a 27 -gauge needle, and the needle was then promptly removed. In sham animals, the same procedure was performed except for the ligation. Finally, the lungs were re-expanded, and the chest wall was closed. The animals remained in a supervised setting until fully conscious.

\section{Echocardiography}

Transthoracic echocardiography was recorded as described previously with an FFsonic 8900 (Fukuda Denshi Co, Tokyo, Japan) equipped with a $13-\mathrm{MHz}$ phased-array transducer at baseline and 4 weeks after TAC or sham surgery ${ }^{18-20}$ Left ventricular internal dimensions at end-systole and end-diastole (LVESD and LVEDD) and interventricular septal wall thickness (IVS) were measured digitally on the M-mode tracings and averaged from at least 3 cardiac cycles ${ }^{18-20}$ Left ventricular fractional shortening (\%LVFS) was calculated as [(LVEDD-LVESD)/LVEDD] $\times 100$.

\section{Morphological Examinations}

At 4 weeks after TAC or sham surgery, mice were killed, coronary arteries were retrogradely flushed with saline, and the heart and lungs were excised and weighed. The heart was fixed with a $10 \%$ solution of formalin in phosphate buffered saline at $4^{\circ} \mathrm{C}$ for $24 \mathrm{~h}$, embedded in paraffin, and then cut serially from the apex to the base.$^{19,20}$ Three sections were stained with hematoxylin-eosin or Masson trichrome stain. Transverse sections were captured digitally, and cardiomyocyte cross-sectional area was measured using a Scion imaging system (Scion Corporation, Frederick, MD, USA) as previously reported 16,19 We traced the outline of at least 200 cardiomyocytes for each section, and the data were averaged.

To assess the degree of fibrosis, the sections stained with Masson trichrome were scanned with computer-assisted videodensitometry, and the images from at least 10 fields for each heart were analyzed as described previously! 19,21 The fibrosis fraction was obtained by calculating the ratio of Masson trichrome-stained connective tissue area (stained blue) to total myocardial area with an image analysis software as described previously 19,21

Separation of Membranous and Cytosolic Fractions and
Western Blotting
Membranous and cytosolic fractions of detergent-ex- tracted PKC were prepared from the left ventricular myocardium as described previously6,22,23 The protein concentration of myocardial samples was carefully determined by assay. Equal amounts of protein extracts from the membranous and cytosolic fractions were loaded on each gel lane, separated with $10 \%$ (sodium dodecyl sulfate-polyacrylamide gel electrophoresis), and transferred to polyvinylidene fluoride membranes. To ensure equivalent protein loading and quantitative transfer efficiency, membranes were stained with Ponceau S before incubating with primary antibody. Subcellular localization of the PKCa isoform was examined by quantitative immunoblotting using an isoformspecific antibody (mouse monoclonal anti-PKCa, Santa Cruz Biotechnology, Inc, Santa Cruz, CA, USA) as reported previously, $6,22,23$ Immunoreactive bands were detected by an ECL kit (Amersham Biosciences Corp, Piscataway, NJ, USA), and a membrane/cytosol ratio of immunoreactivity was used as an index for the extent of PKCa translocation $6,22,23$

\section{Extraction of RNA and Real-Time Reverse Transcriptase- Polymerase Chain Reaction (RT-PCR)}

Total RNA was extracted from the left ventricle using TRIzol (Invitrogen, Tokyo, Japan) and first-strand cDNA was synthesized from $1 \mu \mathrm{g}$ of RNA sample with oligo (dT) primers and superscript II reverse transcriptase as previously described ${ }^{15,19,20}$ To examine mRNA expression levels of atrial natriuretic factor (ANF), $\beta$-MHC, a -MHC, transforming growth factor $\beta_{1}$ (TGF $\beta$ 1), collagen type I, and collagen type III, real-time PCR amplification was performed as reported previously!5,19 Reagents were purchased from Roche Diagnostics Japan (Tokyo, Japan). Amplification was performed using LightCycler DNA Master SYBR Green $I$ in a $20 \mu 1$ volume reaction and analyzed using LightCycler Software Ver.3.5 (Roche Diagnostics Japan). Standard curves of these genes were generated by full sequence plasmid of known concentrations. Gene expressions were normalized to glyceraldehyde 3-phosphate dehydrogenase (GAPDH). Primers were designed based on GenBank sequences (ANF, K02781; $\beta$-MHC, AY056464; a-MHC, M76601; TGF $\beta$ 1, NM011577; collagen type I, NM007742; collagen type III, NM009930; and GAPDH, NM001001303). Sense and anti-sense primers were forward (F) 5'-ACC ACC TGG AGG AGA AGA-3' and reverse (R) 5'-TTC AAG AGG GCA GAT CTA TC-3' for ANF, (F) 5'-GTC CAA GTT CCG CAA GGT-3' and (R) 5'-AGG GTC TGC TGG AGA GGT TA-3' ford-MHC, (F) 5'-CCG AGT CCC AGG TCA ACA A-3' and (R) 5'-CTT CAC GGG CAC CCT TAG A-3' for 3 -MHC, (F) 5'-TGG TAC ATC AGC CCG AAC-3' and (R) 5'-GTC AGC TGG ATA GCG ACA-3' for collagen type I, (F) 5'-GAC AGA TTC TGG TGC AGA GA-3' and (R) 5'-CAT CAA CGA CAT CTT CAG GAAT-3' for collagen type III, (F) 5'-AAC AAC GCC ATC TAT GAG-3' and (R) 5'-TAT TCC GTC TCC TTG GTT-3' for TGF $\beta 1$ and (F) 5'-TGA ACG GGA AGC TCA CTG G-3' and (R) 5'-TCC ACC ACC CTG TTG CTG TA-3' for GAPDH. The reaction conditions for the reverse transcriptase are a denaturing step at $95^{\circ} \mathrm{C}$ for 10 min followed by a 3-step PCR amplification to quantify expression. The steps are: $95^{\circ} \mathrm{C}$ for $10 \mathrm{~s}, 55-62^{\circ} \mathrm{C}$ for $10 \mathrm{~s}$, $72^{\circ} \mathrm{C}$ for $5-9 \mathrm{~s}$ (depending on the gene) for 45 cycles.

\section{Statistical Analysis}

All values are expressed as mean \pm SD. Effects of TAC on heart weight, histological data, echocardiographic mea- 
A

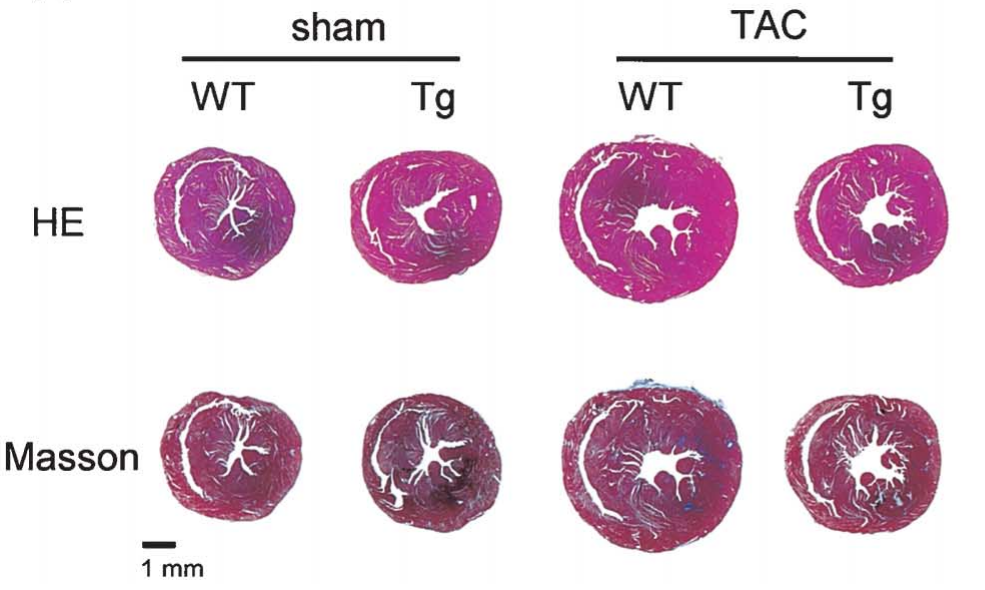

B

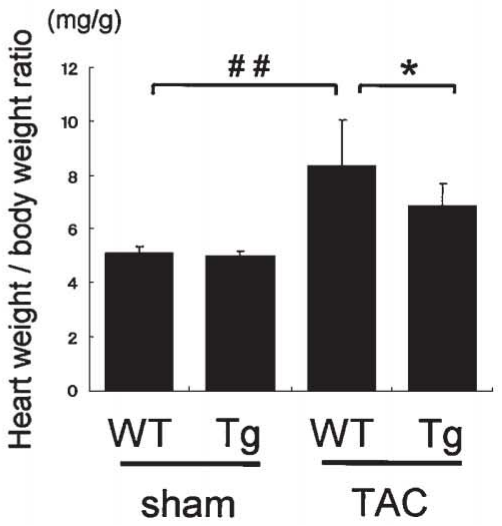

Fig 1. (A) Histological observations of the left ventricular myocardium in WT and DGK -TG mice at 4 weeks after TAC or sham surgery. Left ventricular transverse sections were stained by hematoxylin-eosin or Masson trichrome. (B) Heart weight-to-body weight ratios in WT and DGK -TG mice at 4 weeks after TAC or sham surgery. Data are mean \pm SD from 8 mice for each group. ${ }^{\#} \mathrm{p}<0.01$ vs WT sham and $* \mathrm{p}<0.05$ vs WT TAC. DGK\}, diacylglycerol kinase $\zeta$; TAC, transverse aortic constriction; TG, transgenic; WT, wild type.

A

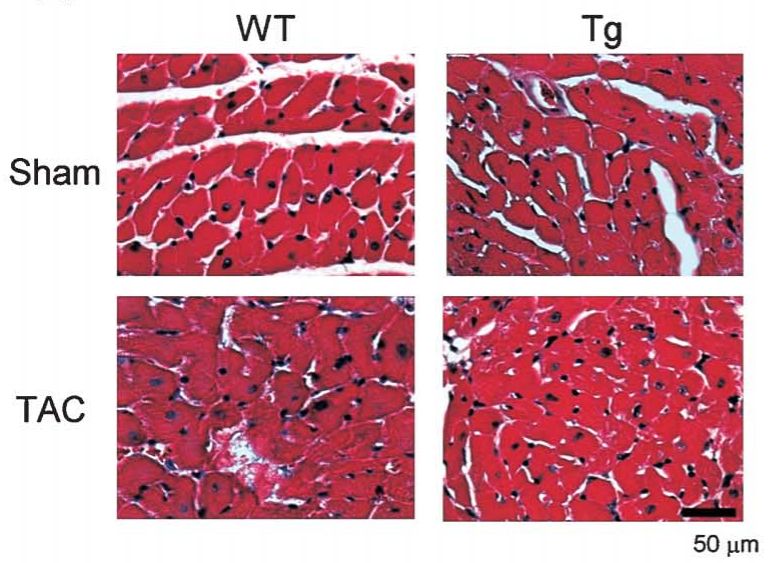

B

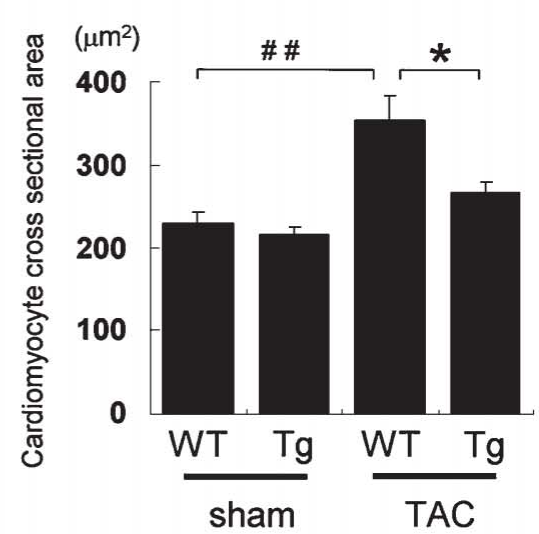

Fig 2. (A) Microscopic observations of hematoxylin-eosin-stained transverse sections of the left ventricular myocardium in WT and DGK -TG mice at 4 weeks after TAC or sham surgery $(\times 400)$. (B) Cardiomyocyte cross-sectional area in WT and DGK -TG mice at 4 weeks after TAC or sham surgery. At least 200 cardiomyocytes were analyzed for each section, and the data were averaged. Data were obtained from 8 mice for each group. ${ }^{\#} \mathrm{p}<0.01$ vs WT sham and $* \mathrm{p}<0.05$ vs WT TAC. See Fig 1 for abbreviations.

surements, and RT-PCR results between WT and TG mice were analyzed by 2-way ANOVA followed by multiple comparisons with a Bonferroni test. A value of $\mathrm{p}<0.05$ was considered statistically significant.

\section{Results}

\section{Cardiac Hypertrophy After TAC}

Fig 1A shows representative transverse sections of DGK TG and WT mouse hearts after sham or TAC surgery. Extensive cardiac hypertrophy was observed at 4 weeks after TAC in WT mice; however, cardiac hypertrophy after TAC was attenuated in DGK -TG mice.

At 4 weeks after surgery, body weight was not different between the 4 groups $(23.9 \pm 2.10 \mathrm{~g}$ for WT-sham, 23.6 \pm $2.44 \mathrm{~g}$ for DGKY $-\mathrm{TG}$-sham, $23.7 \pm 2.58 \mathrm{~g}$ for WT-TAC, and $24.0 \pm 2.38 \mathrm{~g}$ for DGK $-\mathrm{TG}-\mathrm{TAC}$ ), whereas heart weight was markedly increased after TAC surgery $(120.6 \pm 16.7 \mathrm{mg}$ for WT-sham, $120.8 \pm 16.52 \mathrm{mg}$ for DGK\} -TG-sham, 195.1 $19 \mathrm{mg}$ for WT-TAC, and $164.1 \pm 28.5 \mathrm{mg}$ for DGK马 -TGTAC). As shown in Fig 1B, heart weight corrected for body weight was not different between sham-operated WT and sham-operated DGK马 -TG mice at 4 weeks after surgery. At 4 weeks after TAC, the ratio of heart weight to body weight was significantly lower in DGK马 -TG mice than in WT mice.

Microscopic observations revealed no significant difference in cardiomyocyte cross-sectional area in the shamoperated WT mice and sham-operated DGKZ -TG mice (Figs 2A,B). Cardiomyocyte cross-sectional area was increased in WT mice at 4 weeks after TAC $(p<0.01)$. However, increases in cardiomyocyte cross-sectional area after TAC were significantly attenuated in DGKZ -TG mice compared with WT mice $(\mathrm{p}<0.05)$. 
A

sham

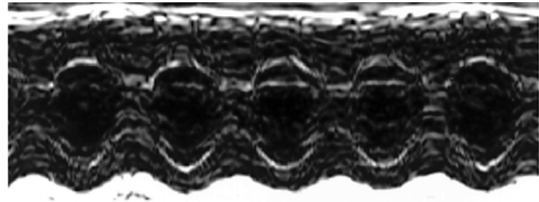

TAC

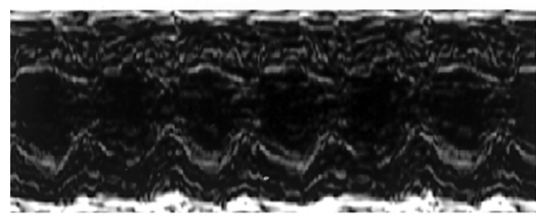

$\mathrm{Tg}$
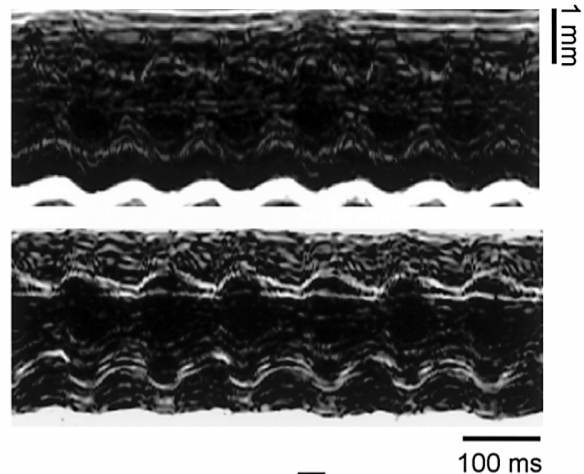

$E$

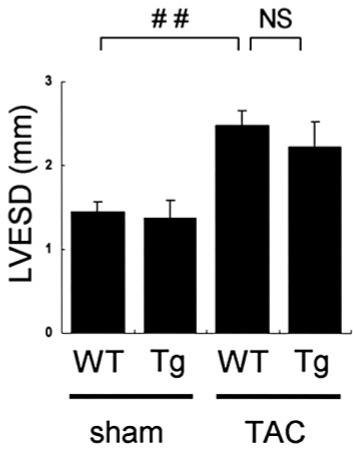

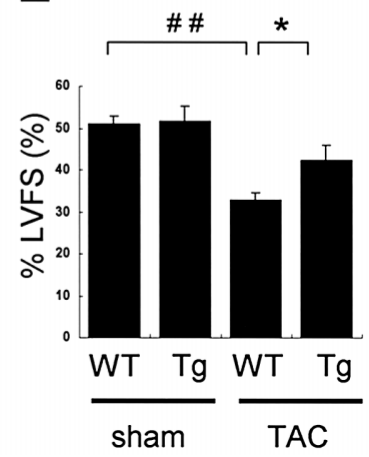

Fig 3. (A) Representative M-mode echocardiograms of WT and DGK -TG mice at 4 weeks after TAC or sham surgery. $(B-E)$ Group data $(n=8)$ of echocardiographic measurements in WT and DGK -TG mice at 4 weeks after TAC or sham surgery. (B) Interventricular wall thickness (IVS); (C) left ventricular end-diastolic dimension (LVEDD); (D) left ventricular end-systolic dimension (LVESD); (E) left ventricular fractional shortening (\%LVFS). ${ }^{\mathrm{p}}<0.05$ and ${ }^{\# \#} \mathrm{p}<0.01$ vs WT sham, ${ }^{*} \mathrm{p}<0.05$ and $* * \mathrm{p}<0.01$ vs WT TAC. See Fig 1 for other abbreviations.

A

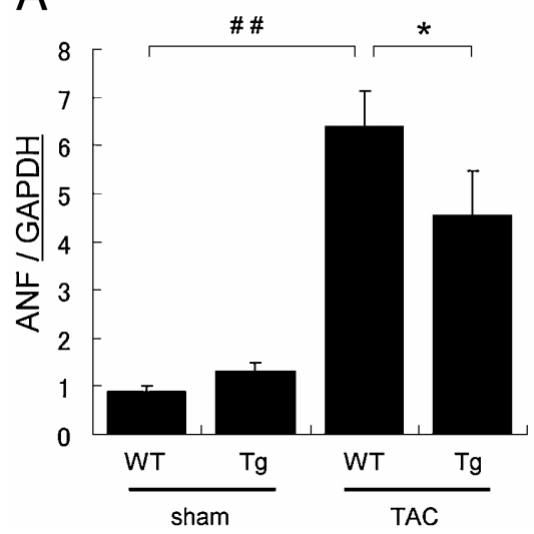

B

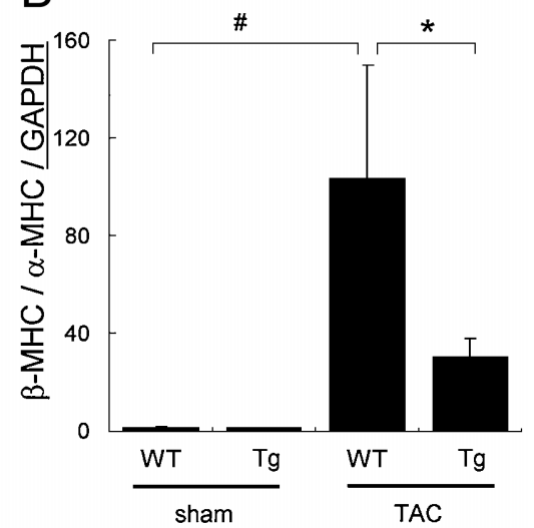

Fig 4. (A) Quantitative analyses of atrial natriuretic factor gene expression by real-time PCR in DGK -TG and WT mice at 4 weeks after TAC or sham surgery. (B) $\beta$-MHC to $\alpha$ MHC ratio by real-time PCR in DGK -TG and WT mice at 4 weeks after TAC or sham surgery. Each expression level was normalized to glyceraldehyde 3-phosphate dehydrogenase (GAPDH). Data are mean \pm SD from 8 mice for each group. ${ }^{*} \mathrm{p}<0.05$ and ${ }^{\# \#} \mathrm{p}<0.01$ vs WT sham, $* \mathrm{p}<0.05$ vs WT TAC. MHC, myosin heavy chain; PCR, polymerase chain reaction. See Fig 1 for other abbreviations.

\section{Echocardiographic Measurements}

Echocardiography was performed at baseline and 4 weeks after TAC or sham surgery in WT and DGK -TG mice. Under anesthesia, the heart rate was similar between WT and DGK马 -TG mice (data not shown). Before surgery, IVS, LVEDD and \%LVFS were similar between WT and DGK -TG mice (data not shown). Representative M-mode echocardiograms at 4 weeks after surgery are shown in Fig 3A. IVS and LVEDD were significantly increased in WT mice after TAC. However, in DGK -TG mice, the increase in IVS after TAC was attenuated compared with the WT mice (Fig 3B). Moreover, the reduction of \% LVFS at 4 weeks after TAC was attenuated in DGK -TG mice compared with WT mice.

\section{Expressions of Fetal Genes in Hypertrophied Hearts}

We next used real-time RT-PCR to examine the mRNA expression of fetal type genes, such as ANF and $\beta-\mathrm{MHC}$, at 4 weeks after TAC in WT and DGK -TG mice (Fig 4). Expressions of ANF were markedly up-regulated in WT mice after TAC. Conversely in DGK -TG mice, gene induction of ANF in response to TAC was significantly attenuated compared with WT mice. The $\beta$-MHC a-MHC ratio was also markedly increased in WT mice after TAC, but this response was blocked in DGK -TG mice. 
A

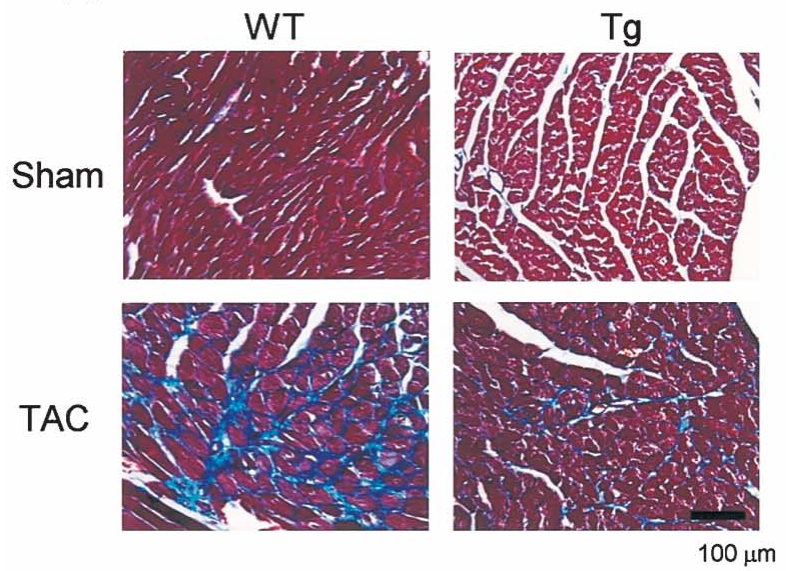

B

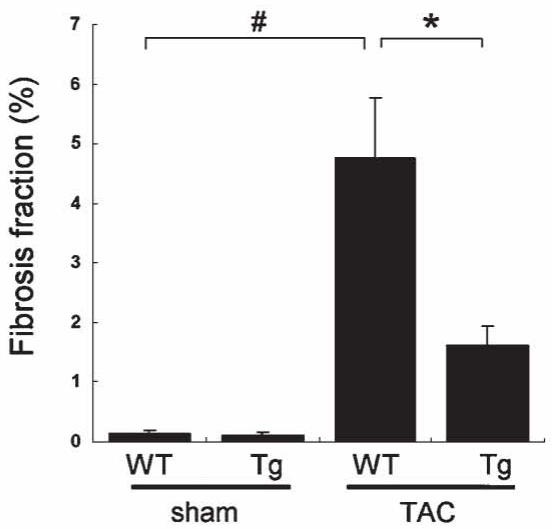

Fig 5. (A) Representative myocardial sections stained with Masson trichrome in WT and DGK -TG mice after TAC or sham surgery $(\times 200)(B)$ Comparisons of the fibrosis fraction between DGK -TG and WT mice. The fibrosis fraction was calculated as the ratio of Masson trichrome stained connective tissue area to total myocardial area. Data are reported as mean \pm SD obtained from 8 mice for each group. ${ }^{\#}<<0.05$ vs WT sham, and $* \mathrm{p}<0.05$ vs WT TAC. See Fig 1 for abbreviations.

A

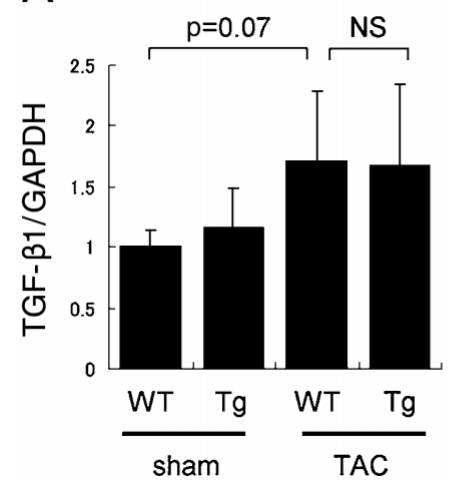

B

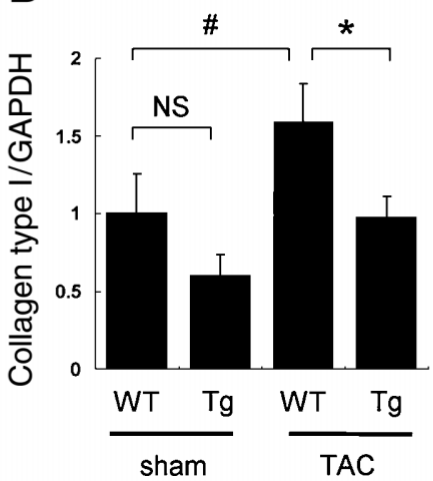

C

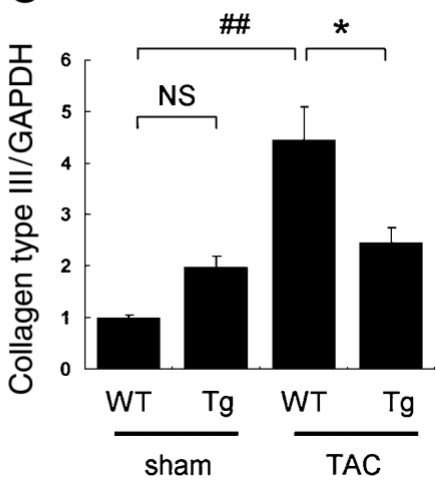

Fig 6. Quantitative analyses of TGF $\beta$ 1 (A), collagen type I (B), and collagen type III (C) gene expressions corrected for glyceraldehyde 3-phosphate dehydrogenase (GAPDH) in WT and DGK -TG mice at 4 weeks after TAC or sham surgery. Data are mean \pm SD from 8 mice for each group. ${ }^{\#} \mathrm{p}<0.05$ and ${ }^{\# \#} \mathrm{p}<0.01$ vs WT sham, ${ }^{*} \mathrm{p}<0.05$ vs WT TAC. TGF, transforming growth factor. See Fig 1 for other abbreviations.
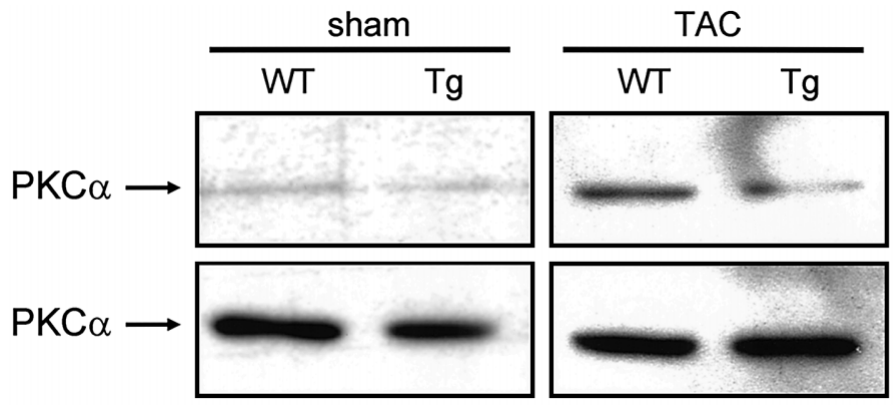

membrane

cytosol
Fig 7. Representative Western blot for subcellular localization of PKCa after TAC in WT and DGKY TG mice. Translocation of the PKCa isoform from cytosolic to membranous fractions was observed in WT mice after TAC. However, the translocation of the PKCa was attenuated in DGK -TG mice after TAC. Similar results were obtained from 3 independent experiments. $\mathrm{PKCa}$, protein kinase $\mathrm{Ca}$ isoform. See Fig 1 for other abbreviations.
Myocardial Fibrosis and Expressions of Profibrotic Genes

Because reactive interstitial and perivascular fibrosis adversely alter myocardial stiffness and ultimately lead to left ventricular dysfunction ${ }^{24}$ we examined myocardial fibrosis at 4 weeks after TAC in WT and DGK -TG mice using sections stained with Masson trichrome (Fig 5A). Prominent fibrosis was observed in WT mice at 4 weeks after TAC as reported previously? ${ }^{25}$ However, the degree of myocardial fibrosis was much less in DGK -TG mice than in WT mice at 4 weeks after TAC (Figs 5A,B). These results indicated that, although pressure overload caused fibrous formation in the left ventricular myocardium, reactive fibrosis was significantly attenuated by DGK .

We next examined the expression of profibrotic genes, such as TGF $\beta$ 1, collagen type I, and collagen type III, to investigate whether these morphological observations were 
accompanied by alterations in gene expressions relevant to fibrotic changes 26,27 As shown in Fig 6, real-time PCR revealed that TGF $\beta$, collagen type I, and collagen type III mRNA at 4 weeks after TAC were up-regulated in WT mice. However, these increases in collagen types I and III mRNA, but not TGF $\beta$, were significantly attenuated in DGK -TG mice compared with WT mice.

\section{Translocation of $\mathrm{PKCa}$}

Because we reported that DGK blocks activation of PKC isoforms in response to GPCR agonists, we next examined translocation of the PKC isoforms in DGK -TG and WT mouse hearts after TAC. As shown in Fig 7, we detected translocation of the PKCa isoform from the cytosolic fraction to the membranous fraction in the hypertrophied WT mouse heart at 4 weeks after TAC. However, in DGK -TG mice, translocation of PKCa after TAC was attenuated.

\section{Discussion}

In the present study, we demonstrated that pressure-overload-induced cardiac hypertrophy and left ventricular systolic dysfunction were attenuated in transgenic mice with cardiac specific overexpression of DGK (DGK -TG) compared with their WT littermates. We also showed that cardiac fibrosis and induction of profibrotic genes by pressure overload were blocked in DGK -TG mice.

Cardiac hypertrophy is an initially adaptive response to several forms of cardiac disease, but sustained hypertrophy is a powerful independent risk factor for cardiac morbidity and mortality!,2 Many lines of experimental and clinical evidence have suggested the importance of the Gca-phosphoinositide signaling system in the development of pathologic cardiac hypertrophy and heart failure ${ }^{3-7}$ GPCR agonists, such as angiotensin II, endothelin-1, and phenylephrine, induce cardiac hypertrophy in part via the activation of several PKC isoforms. We have recently demonstrated that this activation of PKC isoforms is DAG dependent and overexpression of DGK , one major terminator for DAG signaling, inhibits the translocation of PKCa and PKCE, leading to the prevention of concomitant cardiac hypertrophy ${ }^{15,16}$ However, the functional role of DGKS in hemodynamic-overload-induced changes in cellular signaling, cardiac structure and function has not been previously examined. Because mechanical stimuli such as pressure and volume overload are clinically relevant and important ${ }^{28}$ in the present study we examined whether DGK modifies cardiac remodeling in response to pressure overload using a TAC mouse model. We showed that cardiac hypertrophy by TAC was attenuated in DGK -TG mice compared with WT mice. Impaired left ventricular systolic function at 4 weeks after TAC was prevented in DGK -TG mice, suggesting that progression from compensated cardiac hypertrophy to decompensated heart failure might be delayed or prevented by DGK .

Excessive myocardial fibrosis is an important pathological process contributing to diastolic and eventually systolic left ventricular dysfunction in hypertrophied hearts ${ }^{24}$ Pressure-overload-induced cardiac fibrosis causes left ventricular dysfunction resulting from the increased myocardial stiffness and reduced pumping capacity. 9,30 In the present study, DGK 3 blocked pressure-overload-induced cardiac fibrosis and expression of pro-fibrotic genes such as TGF$\beta_{1}$, collagen type I and type III in vivo. TGF $\beta 1$ has been reported to be expressed, particularly during the transition from hypertrophy to heart failure, in experimental animal models $^{31}$ and human heart failure ${ }^{32}$ and is a marker discriminating between compensated and decompensated cardiac hypertrophy. It has been reported that valsartan, an angiotensin II type 1 receptor blocker, decreases type I collagen synthesis in patients with hypertrophic cardiomyopathy, indicating the antifibrotic effects of inhibiting GPCR signaling cascade 33

It has been reported that PKCa, the dominant PKC isoform in rabbit and mouse cardiac muscle, reduces cardiac contractility and negatively regulates ventricular systolic and diastolic function in pathological hypertrophy 34,35 Transgenic overexpression of PKCa causes a lethal restrictive cardiomyopathy with marked interstitial fibrosis 34 Deletion of PKCa exhibits hypercontractility and protects against cardiac fibrosis and heart failure by modulating calcium handling proteins ${ }^{35}$ Therefore, the inhibition of PKCa translocation by DGK (Fig 7) might be a possible mechanism for the preservation of left ventricular function, attenuation of fibrosis, and blockade of pro-fibrotic gene induction observed in the present study 36 It remains to be elucidated whether calcium handling proteins, such as sarcoplasmic reticulum calcium ATPase 2a or phospholamban, are actually affected by DGK inorder to account for the mechanisms of the beneficial effects of PKCa inhibition. Our present data showing inhibition of PKCa translocation by DGK are supported by an in vitro study demonstrating a spatial association of DGK马 with PKCa in HEK293 cells? ${ }^{37}$

\section{Conclusions}

We demonstrated that DGK 3 attenuated cardiac hypertrophy and fibrosis in response to pressure overload and prevented subsequent left ventricular systolic dysfunction in vivo. These results suggest a potential novel therapeutic target to prevent cardiac structural remodeling in response to mechanical overload.

\section{Acknowledgments}

This study was supported in part by a grant-in-aid for Scientific Research (No. 17590702) from the Ministry of Education, Science, Sports and Culture, Japan, a grant-in-aid from the 21st Century Center of Excellence (COE) Program of the Japan Society for the Promotion of Science, and grants from Takeda Science Foundation and Fukuda Foundation for Medical Technology.

\section{References}

1. Walsh RA. Molecular mechanisms for cardiac hypertrophy and failure. In: Walsh RA, editor. Taylor \& Francis. London, UK; 2005; 746.

2. Levy D, Garrison RJ, Savage DD, Kannel WB, Castelli WP. Prognostic implications of echocardiographically determined left ventricular mass in the Framingham Heart Study. N Engl J Med 1990; 322: $1561-1566$.

3. Sugden PH, Clerk A. Cellular mechanisms of cardiac hypertrophy. J Mol Med 1998; 76: 725-746.

4. Molkentin JD, Dorn GW. Cytoplasmic signaling pathways that regulate cardiac hypertrophy. Annu Rev Physiol 2001; 63: 391-426.

5. Oyama N, Urasawa K, Kaneta S, Sakai H, Saito T, Takagi C, et al. Chronic beta-adrenergic receptor stimulation enhances the expression of G-Protein coupled receptor kinases, GRK2 and GRK5, in both the heart and peripheral lymphocytes. Circ J 2005; 69: 987 990 .

6. Takeishi Y, Chu G, Kirkpatrick DM, Li Z, Wakasaki H, Kranias EG, et al. In vivo phosphorylation of cardiac troponin I by protein kinase Cbeta2 decreases cardiomyocyte calcium responsiveness and contractility in transgenic mouse hearts. J Clin Invest 1998; 102: 72-78.

7. Bowling N, Walsh RA, Song G, Estridge T, Sandusky GE, Fouts RL, 
et al. Increased protein kinase $\mathrm{C}$ activity and expression of $\mathrm{Ca} 2+-$ sensitive isoforms in the failing human heart. Circulation 1999; 99: 384-391.

8. Takeishi Y, Ping P, Bolli R, Kirkpatrick DL, Hoit BD, Walsh RA. Transgenic overexpression of constitutively active protein kinase $\mathrm{C}$ epsilon causes concentric cardiac hypertrophy. Circ Res 2000; 86: $1218-1223$.

9. Wang J, Liu X, Sentex E, Takeda N, Dhalla NS. Increased expression of protein kinase $\mathrm{C}$ isoforms in heart failure due to myocardial infarction. Am J Physiol Heart Circ Physiol 2003; 284: H2277$\mathrm{H} 2287$.

10. Sakane F, Yamada K, Kanoh H, Yokoyama C, Tanabe T. Porcine diacylglycerol kinase sequence has zinc finger and $\mathrm{E}-\mathrm{F}$ hand motifs. Nature 1990; 344: 345-348.

11. Goto K, Kondo H. A 104-kDa diacylglycerol kinase containing ankyrin-like repeats localizes in the cell nucleus. Proc Natl Acad Sci USA 1996; 93: 11196-11201.

12. Topham MK, Bunting M, Zimmerman GA, McIntyre TM, Blackshear PJ, Prescott SM. Protein kinase C regulates the nuclear localization of diacylglycerol kinase-zeta. Nature 1998; 394: 697-700.

13. Goto K, Kondo H. Diacylglycerol kinase in the central nervous system: Molecular heterogeneity and gene expression. Chem Phys Lipids 1999; 98: 109-117.

14. Takeda M, Kagaya Y, Takahashi J, Sugie T, Ohta J, Watanabe J, et al. Gene expression and in situ localization of diacylglycerol kinase isozymes in normal and infarcted rat hearts: Effects of captopril treatment. Circ Res 2001; 89: 265-272.

15. Takahashi H, Takeishi Y, Seidler T, Arimoto T, Akiyama H, Hozumi $\mathrm{Y}$, et al. Adenovirus-mediated overexpression of diacylglycerol kinase-zeta inhibits endothelin-1-induced cardiomyocyte hypertrophy. Circulation 2005; 111: 1510-1516.

16. Ariomoto T, Takeishi Y, Takahashi H, Shishido T, Niizeki T, Koyama $Y$, et al. Cardiac-specific overexpression of diacylglycerol kinase $\zeta$ prevents $\mathrm{Gq}$ protein-coupled receptor agnonists-induced cardiac hypertrophy in transgenic mice. Circulation 2006 (in press).

17. Esposito G, Prasad SVN, Rapacciuolo A, Mao L, Koch WJ, Rockman HA. Cardiac overexpression of a $\mathrm{Gq}$ inhibitor blocks induction of extracellular signal-regulated kinase and c-Jun NH2-terminal kinase activity in vivo pressure overload. Circulation 2001; 103: 1453 1458.

18. Gao XM, Dart AM, Dewar E, Jennings G, Du XJ. Serial echocardiographic assessment of left ventricular dimensions and function after myocardial infarction in mice. Cardiovasc Res 2000; 45: 330-338.

19. Shishido T, Nozaki N, Yamaguchi S, Shibata Y, Nitobe J, Miyamoto $\mathrm{T}$, et al. Toll-like receptor-2 modulates ventricular remodeling after myocardial infarction. Circulation 2003; 108: 2905-2910.

20. Nozaki N, Shishido T, Takeishi Y, Kubota I. Modulation of doxorubicin-induced cardiac dysfunction in toll-like receptor-2-knockout mice. Circulation 2004; 110: 2869-2874.

21. Weber KT, Brilla CG. Pathological hypertrophy and cardiac interstitium: Fibrosis and renin-angiotensin-aldosterone system. Circulation 1991; 83: $1849-1865$.

22. Takeishi Y, Jalili T, Ball NA, Walsh RA. Responses of cardiac pro- tein kinase $\mathrm{C}$ isoforms to distinct pathological stimuli are differentially regulated. Circ Res 1999; 85: 264-271.

23. Takahashi H, Takeishi Y, Miyamoto T, Shishido T, Arimoto T, Konta $\mathrm{T}$, et al. Protein kinase $\mathrm{C}$ and extracellular signal regulated kinase are involved in cardiac hypertrophy of rats with progressive renal injury. Eur J Clin Invest 2004; 34: 85-93.

24. Kuwahara F, Kai H, Tokuda K, Takeya M, Takeshita A, Egashira K, et al. Hypertensive myocardial fibrosis and diastolic dysfunction. Hypertension 2004; 43: 739-745.

25. Takimoto E, Champion HC, Li M, Ren S, Rodriguez ER, Tavazzi B, et al. Oxidant stress from nitric oxide synthase-3 uncoupling stimulates cardiac pathologic remodeling from chronic pressure load. $J$ Clin Invest 2005; 115: $1221-1231$.

26. Lijnen PJ, Petrov VV, Fagard RH. Induction of cardiac fibrosis by transforming growth factor $\beta 1$. Mol Genet Metab 2000; 71: 418435.

27. Manabe I, Shindo T, Nagai R. Gene expression in fibroblasts and fibrosis: Involvement in cardiac hypertrophy. Circ Res 2002; 91: $1103-1113$.

28. Miyamoto T, Takeishi Y, Takahashi H, Shishido T, Arimoto T, Tomoike $\mathrm{H}$, et al. Activation of distinct signal transduction pathways in hypertrophied hearts by pressure and volume overload. Basic Res Cardiol 2004; 99: 328-337.

29. Gaasch WH, Zile MR. Left ventricular diastolic dysfunction and diastolic heart failure. Anпи Rev Med 2004; 55: 373-394.

30. Gillebert TC, Leite-Moreira AF, De Hert SG. Load dependent diastolic dysfunction in heart failure. Heart Fail Rev 2000; 5: 345-355.

31. Bolyut MO, O'Neill L, Meredith AL, Bing OH, Brooks WW, Conrad $\mathrm{CH}$, et al. Alterations in cardiac gene expression during the transition from stable hypertrophy to heart failure: Marked upregulation of genes encoding extracellular matrix components. Circ Res 1994; 75: 23-32.

32. Song H, Foster AH, Conte JV, Chi-Ming W. Presentation and localization of transforming growth factor beta isoforms and its receptor subtypes in human myocardium in the absence and presence of heart failure. Circulation 1997; 96(Suppl I): I-362.

33. Kawano H, Toda G, Nakamizo R, Koide Y, Seto S, Yano K. Valsartan decreases type I collagen synthesis in patients with hypertrophic cardiomyopathy. Circ J 2005; 69: 1244-1248.

34. Hahn HS, Marreez Y, Odley A, Sterbling A, Yussman MG, Hilty $\mathrm{KC}$, et al. Protein kinase $\mathrm{C}$ alpha negatively regulates systolic and diastolic function in pathological hypertrophy. Circ Res 2003; 93: $1111-1119$

35. Braz JC, Gregory K, Pathak A, Zhao W, Sahin B, Klevitsky R, et al. PKC-alpha regulates cardiac contractility and propensity toward heart failure. Nat Med 2004; 10: 248-254.

36. Boyle AJ, Kelly DJ, Zhang Y, Cox AJ, Gow RM, Way K, et al. Inhibition of protein kinase $\mathrm{C}$ reduces left ventricular fibrosis and dysfunction following myocardial infarction. J Mol Cell Cardiol 2005; 39: 213-221.

37. Luo B, Prescott SM, Topham MK. Association of diacylglycerol kinase zeta with protein kinase $\mathrm{C}$ alpha: Spatial regulation of diacylglycerol signaling. J Cell Biol 2003; 160: 929-937. 